\title{
Cadernos de Estudos Africanos
}

21 | 2011

Varia

\section{Entre Deux Drapeaux: Les ouvriers capverdiens au Portugal pendant la période revolutionnaire (1974-1976)}

\section{Antoine Acker}

\section{(2) OpenEdition Journals}

Édition électronique

URL : http://journals.openedition.org/cea/219

DOI : 10.4000/cea.219

ISSN : 2182-7400

Éditeur

Centro de Estudos Internacionais

\section{Édition imprimée}

Date de publication : 1 octobre 2011

Pagination : 123-145

ISSN : 1645-3794

\section{Référence électronique}

Antoine Acker, « Entre Deux Drapeaux: Les ouvriers capverdiens au Portugal pendant la période revolutionnaire (1974-1976) », Cadernos de Estudos Africanos [En ligne], 21 | 2011, mis en ligne le 22 juillet 2012, consulté le 01 mai 2019. URL : http://journals.openedition.org/cea/219 ; DOI : 10.4000/ cea. 219

\section{cc) (i) (2) (2)}

O trabalho Cadernos de Estudos Africanos está licenciado com uma Licença Creative Commons Atribuição-NãoComercial-Compartilhalgual 4.0 Internacional. 


\section{Entre Deux Drapeaux: Les ouvriers capverdiens au Portugal pendant} LA PÉRIOdE REVOLUTIONNAIRE (1974-1976)

Antoine Acker

Department of History and Civilization (HEC) European University Institute (EUI), Florence antoine.acker@eui.eu 


\section{Résumé}

Marqués par un climat de décolonisation, les mois qui suivirent la Révolution des Oeillets (25 avril 1974) constituèrent une période d'incertitude pour les Capverdiens vivant et travaillant en tant qu'ouvriers au Portugal. Axé sur cet interstice historique de création d'identités post-coloniales (1974-1976), cet article s'interroge sur la marge d'action des ouvriers capverdiens vivant entre deux nations (le Portugal et le Cap Vert) encore à (ré)inventer. Ainsi, l'article s'intéresse aux politiques et aux comportements adoptés par les acteurs sociaux portugais et capverdiens à l'encontre des migrants, mais aussi aux stratégies des migrants eux-mêmes face à l'incertitude, aux crises, et aux phénomènes d'exclusion qui eurent lieu durant la période révolutionnaire.

Mots-clé: décolonisation, révolution, indépendance, migrants, ouvriers du bâtiment, Cap Vert

\section{Resumo}

Marcados por um contexto de descolonização, os meses que se seguiram à Revolução dos Cravos (25 de Abril de 1974) constituíram um período de incerteza para os cabo-verdianos que viviam e trabalhavam em Portugal como operários. Centrado nesse interstício histórico de construção de identidades pós-coloniais (1974-1976), este artigo interroga-se sobre a margem de manobra dos trabalhadores cabo-verdianos a viver entre duas nações (Portugal e Cabo Verde) ainda por (re)inventar. O artigo toma em consideração as políticas e os comportamentos adoptados pelos actores sociais portugueses e cabo-verdianos em relação aos imigrantes, mas também as estratégias dos próprios imigrantes face à incerteza, às crises e aos fenómenos de exclusão que tiveram lugar durante o período revolucionário.

Palavras-chave: descolonização, revolução, independência, imigrantes, trabalhadores da construção civil, Cabo Verde 
Entre deux drapeaux: c'est la traduction du titre d'un roman de l'écrivain capverdien Henrique Teixeira de Sousa (1994). L'action se déroule au Cap Vert, dans une période d'incertitude aussi bien institutionnelle qu'identitaire, entre la chute du régime autoritaire portugais (25 avril 1974) et l'indépendance de l'archipel (5 juillet 1975). Si la métaphore de Teixeira de Sousa épouse habilement la chronologie historique, elle est aussi l'expression d'une position fragile, entre la perte des repères liée à la liquidation du vieil empire portugais, et les conflits qui pèsent sur les choix d'avenir. Entre deux drapeaux, c'est sans doute la juste expression pour désigner les sentiments mêlés de précarité et d'enthousiasme, de peur du déclassement et de foi en l'avenir, de nostalgie et de désir d'en découdre avec le passé, qui divisent les différents protagonistes du roman. Entre deux drapeaux, c'est l'expression d'identités ballottées, hésitantes, en mutation, qui pourrait s'adapter aussi bien aux ouvriers capverdiens vivant ou travaillant au Portugal à la même époque. Au lendemain de la révolution d'avril, ces ouvriers devaient faire face à l'incertitude quant au devenir de leur statut et de leur nationalité, à la violente crise frappant le secteur du bâtiment, et au déclenchement d'une vague d'hostilité à leur encontre exprimée par des acteurs portugais et capverdiens. Tout ceci en faisait des individus positionnés entre deux drapeaux, sans garantie d'intégration, que ce soit dans le nouveau paysage portugais ou dans la nation capverdienne naissante.

L'immigration de milieux populaires capverdiens au Portugal commença à atteindre des proportions massives à la fin des années 19601․ Il s'agissait en majorité de paysans de l'île de Santiago, la plus pauvre de l'archipel, encouragés par les réseaux du régime colonial à gagner la métropole. Leur arrivée avait été réclamée par les syndicats patronaux portugais. Elle devait pallier la fuite massive des ouvriers non qualifiés du pays vers le nord de l'Europe, en particulier dans le domaine de la construction civile, le bâtiment, mais aussi dans d'autres secteurs comme les mines ou les chantiers navals². Malgré l'absence de données fiables de la part des organes statistiques de l'époque cette immigration a été estimée par divers auteurs à plusieurs dizaines de milliers - sans qu'il soit possible d'avancer un chiffre exact (Cf. Batalha, 2004, p. 87; Carreira, 1977, p. 265; Vaz, 1972, p. $8)^{3}$. Elle supplanta en quelques années une autre forme d'immigration capverdienne, plus réduite mais plus privilégiée, de commerçants, de propriétaires, de

\footnotetext{
1 Pour une représentation détaillée de ce point et des suivants, voir Acker, 2009.

2 Si les immigrés étaient en grande majorité des hommes, il existait aussi une immigration capverdienne féminine plus restreinte, notamment dans les secteurs des services aux personnes. Seuls les débuts du regroupement familial dans les années 1970 commencèrent à modifier cette tendance de déséquilibre entre les genres (Cf. Acker, 2009).

3 Pour une analyse détaillée des chiffres de l'Institut National de la Statistique (INE) portugais ainsi que des spéculations développées dans la littérature, voir Acker, 2009.
} 
fonctionnaires et d'étudiants, implantée au Portugal depuis des décennies (Cf. Saint-Maurice, 1997, p. 49). On retrouvait cette immigration plus ancienne dans la sociologie des membres ainsi que les centres d'intérêts de la Casa de Cabo Verde (CCV), seule instance de représentation des Capverdiens avant 1974, alignée sur les recommandations du régime colonial. A l'image des membres de la Casa, les ressortissants de cette immigration en quelque sorte privilégiée étaient en général dotés d'un haut niveau de formation et issus des parties plus riches de l'archipel, en particulier l'île de São Vicente, dont la population était considérée par le régime colonial comme la plus européanisée.

Cet article ne prétend par retracer dans son entier l'émergence, la consolidation et la diversité de l'immigration capverdienne au Portugal. Il s'arrête sur un moment historique de transition: la période de révolution et de décolonisation des années 1974, 1975 et 1976, entre la chute du régime de l'Etat Nouveau et l'avènement de la nouvelle constitution portugaise qui, en reconnaissant "le droit des peuples à l'autodétermination et à l'indépendance [...] tout comme le droit à l'insurrection contre toutes les formes d'oppression", administra le coup de grâce au rêve impérial lusitanien (Constituição da República Portuguesa. Lei do Tribunal Constitucional, 2005, art. 7, al. 3). Il s'agit donc d'une période d'entre-deux, de recomposition des nations portugaise et africaines, dans le cadre de laquelle les identités hybrides, trans-continentales et post-coloniales méritent un intérêt particulier. Par ailleurs cet article est consacré essentiellement à une catégorie spécifique de migrants: ceux que l'on classifie traditionnellement sous l'appellation d'immigration économique, venus au Portugal à la recherche d'emplois et recrutés dans les secteurs manuels et non qualifiés. Dans un souci de synthèse plus que d'exactitude, on utilisera le terme d'ouvriers.

Arrivés dans les centres urbains de l'ancienne métropole en tant que Portugais d'outre-mer, ces ouvriers virent leur identité coloniale remise en question avec le 25 avril 1974. Leur devenir dans le cadre de la nation capverdienne à construire était aussi incertain que leur position dans la démocratie portugaise en chantier. À l'heure où se fissuraient les identités coloniales figées, quel espace peut-on identifier pour des individus entre deux drapeaux, quelle place dans les institutions, parmi les acteurs sociaux et dans les projets nationaux ou trans-nationaux? Cette interrogation est l'objet de notre article qui, en abordant la situation des ouvriers capverdiens dans un contexte de transition post-coloniale et pré-démocratique, s'interroge sur la marge d'action de ces immigrés. D'ores et déjà, l'on peut dire que la révolution d'avril ne mit pas fin aux relations de domination, aux préjugés coloniaux ou aux discriminations des ressortissants africains sur les lieux de travail. L'indépendance du Cap Vert ne représenta pas non plus l'avènement d'une 
nouvelle entité protectrice pour les immigrés. Pour autant, il serait réducteur de cantonner ces derniers dans une attitude de passivité. Sans nier les tentatives de manipulation dont ils firent l'objet, nous voulons aussi montrer leur résistance face à la crise économique et sociale, leurs stratégies pour faire remonter les revendications, leur participation à la construction d'identités collectives post-coloniales, que ce soit dans le cadre de la démocratisation sociale et politique du Portugal ou dans celui de l'élaboration de la nation capverdienne.

\section{Les associations}

Les conséquences de la révolution du 25 avril 1974 s'illustrèrent rapidement parmi les Capverdiens au Portugal. Début mai, les étudiants capverdiens "prirent d'assaut" la Casa de Cabo Verde pour en débarquer la direction (Cf. Lopes, 2002, p. 4). Une commission fut chargée de purger ses statuts des clauses héritées du régime colonial. Un groupe constitué essentiellement d'étudiants et de syndicalistes entreprit d'orienter la Casa en direction de l'intérêt des travailleurs immigrés et de l'activisme politique (Cf. Carita \& Rosendo, 1993, pp. 143-144). Proches d'un groupement très politisé, le GADCG, ce groupe finit par noyauter la CCV au point d'obtenir, en 1976, la fusion des deux organisations dans une nouvelle association, 1'ACVG (Association de Capverdiens et Guinéens), nettement plus favorable au PAIGC.

Pur produit de la révolution du 25 avril, le GADCG fut créé le 12 mai 1974 sous le nom de GADC (Groupement d'Action Démocratique Capverdien) par une centaine de militants (Presença Cabo-verdiana ${ }^{4}, 1974$, novembre, p. 12). En vue d'une "action intensive de politisation du peuple capverdien", l'organisation décida la constitution de commissions locales dans tous les lieux où existaient des noyaux de population immigrée. Le GADC s'illustrait par un soutien appuyé à la politique du Parti de l'Indépendance de la Guinée et du Cap Vert (PAIGC, le parti dominant sur l'archipel à partir de la fin de l'Estado Novo). Il cherchait à en faire connâ̂tre l'histoire, les personnalités et les grands principes auprès du public émigré (Diário Popular, 17/6/74, p. 9). En ajoutant à la fin de son sigle un G, pour Guinée, quelques heures seulement après sa fondation, le GADCG s'était mis en conformité avec les principes du PAIGC, qui se voulait un parti transnational (Lopes, 2002, p. 294).

Les activités du groupe ne se limitaient pas à porter la parole indépendantiste. Il multiplia les “brigades d'alphabétisation" formées par les étudiants dans

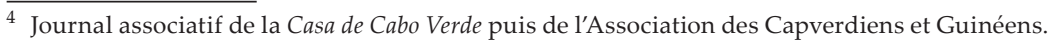


les communautés ouvrières immigrées (Nôs $L u t a^{5}, 1 / 6 / 75$, p. 8). Aspirant au rôle d'interface entre les migrants et le reste de la société, le GADCG s'était également donné pour mission d'alerter l'opinion portugaise à propos des discriminations subies par les travailleurs africains (ibid.).

Les préoccupations du GADCG semblaient donc largement plus tournées qu'autrefois la CCV vers le milieu des travailleurs. Doit-on pour autant en déduire que la mort de cette dernière engendra une démocratisation de la vie associative capverdienne en faveur des ouvriers? En se basant sur une comparaison des fichiers de membres de la CCV et de l'ACVG, Cristina Carita et Vasco Rosendo croient identifier une substitution de "l'élite dominante", c'est à dire des "cadres supérieurs, techniciens et fonctionnaires publics et coloniaux", par une "majorité d'ouvriers et de travailleurs de la production" (1993, p. 147 - ma traduction). Les deux auteurs parlent de "désélitisation" des activités culturelles de l'association, soulignent sa nouvelle politique d'intervention sociale et la réalisation enfin concrétisée d'activités de soutien aux travailleurs. Alcestina Tolentino, ancienne présidente de l'Association Capverdienne de Lisbonne, héritière de l'ACVG, affirme encore plus directement que dans la foulée du 25 avril les travailleurs émigrés se seraient approprié l'espace associatif pour en transformer le profil et modifier les objectifs (Cit. in Raimundo, 2008, p. 338).

Il est vrai qu'avec la transformation de la CCV en ACVG, le nombre d'adhérents ouvriers fit plus que doubler. Mais il était suivi de près par celui des étudiants, nettement surreprésentés par rapport à leur poids réel dans l'ensemble de la communauté capverdienne (Cf. Carita \& Rosendo, 1993, p. 148). Ces étudiants n'hésitaient pas à parler au nom des travailleurs (Presença Cabo-verdiana, 7/8/74, p. 10) et à en revendiquer unilatéralement le soutien (Nôs Luta, 1/6/75, p. 8).

En fait de désélitisation de la structure associative, il conviendrait de parler d'une nouvelle forme d'élitisation, privilégiant les noyaux les plus instruits, les plus syndiqués, les plus proches du PAIGC ou les plus implantés dans le paysage politique portugais. La hiérarchie associative devenait informelle, entre d'un côté l'avant-garde qui portait la lutte politique, et de l'autre les masses laborieuses devant encore être éclairées. Les représentants du GADCG firent leur le mot d'ordre du PAIGC d'après lequel "celui qui sait une chose, qu'il l'enseigne à celui qui ne sait rien", revendiquant son application auprès des travailleurs immigrés dans les comités locaux (Cit. in ibid. - ma traduction). L'hypothèse selon laquelle les ouvriers pouvaient apporter des connaissances aux étudiants n'était pas même soulevée. Il restait donc du chemin à parcourir pour réduire le fossé entre les élites et les ouvriers.

\footnotetext{
${ }^{5}$ Publication capverdienne favorable au PAIGC.
} 
En revanche la fin du monopole de la CCV fut un véritable facteur de démocratisation associative. On tissa localement les premières mailles d'un filet qui, quelques années plus tard, fut en mesure de couvrir une partie importante de la population capverdienne en métropole. Parmi les initiatives les plus créatives, on peut citer l'apparition en juillet 1974 du Cretcheu Futebol Clube, constitué par des Capverdiens employés sur les chantiers navals de la rive sud du Tage. Cette association à vocation sportive au départ, s'illustra très vite sur toutes sortes de terrains, de l'animation populaire à l'organisation d'activités pour les enfants d'immigrés (Cf. Raimundo, 2008, pp. 384-385). Il faut aussi relever le lancement par le syndicaliste Manuel Correia de la Comissão de apoio aos récem-chegados qui, face à l'explosion des flux migratoires et en particulier ceux de 1976 où la plupart des arrivants débarquèrent sans papiers, chercha à mettre à profit, dans une structure d'assistance et de conseil aux nouveaux arrivants, l'expérience des immigrés déjà installés (Cf. Rocha, Agualusa, \& Semedo, 1993, p. 62). Les Comissões de Moradores, associations autogestionnaires qui fleurirent dans les quartiers populaires à l'occasion de la période révolutionnaire, constituèrent également une opportunité pour les Capverdiens de s'impliquer dans la vie locale et s'engager aux côtés de leurs voisins portugais (Cf. Monteiro, 1995, p. 55). Beaucoup y firent leurs premières armes, s'appuyant sur cette expérience pour, dans les années 1980, participer à la construction d'une vie associative plus diversifiée, plus influente et mieux répartie sur le territoire urbain.

\section{Les partis politiques capverdiens}

Parallèlement au développement de l'engagement associatif, le 25 avril avait entraîné la généralisation de bouleversements politiques parmi les Capverdiens de la métropole. Le PAIGC faisait fonctionner une structure métropolitaine menant un travail de propagande auprès des milieux ouvriers. Il concentrait ses efforts sur la promotion des trois points de son programme qui rencontraient le plus de réticence: l'indépendance totale de l'archipel, l'opposition intransigeante à la tenue d'un référendum concernant ce point, et l'union du nouvel État avec celui de la Guinée Bissau (Diário de Lisboa, 16/8/74, pp. 1, 20). Les thèses du PAIGC ne faisaient pas l'unanimité dans les couches populaires des Capverdiens de la métropole. Certains craignaient que l'alliance avec la Guinée ne se solde par le renoncement à la souveraineté nationale de l'archipel (ibid.; ibid. 5/7/75, II, Supplément). D'autres voulaient croire que l'indépendance, conduite par le PAIGC, conduirait à une soumission du pays aux ordres de l'URSS (Presença Caboverdiana, $7 / 8 / 74$, p. 8). 
Jorge Fonseca, un Portugais possédant des attaches au Cap Vert, s'imposa comme le chef de file de l'opposition métropolitaine au PAIGC (A Capital, 19/8/74, p. 13). Il dirigeait l'antenne portugaise de l'Union Démocratique Capverdienne (UDC), l'un des principaux partis d'opposition au PAIGC. Bien qu'officiellement anticolonialiste, ce parti réunissait aussi bien des démocrates mobilisés contre les dérives autoritaristes du PAIGC que des nostalgiques du colonialisme voyant dans l'UDC un outil pour stopper l'engrenage indépendantiste. Du coup, les militants, pour la plupart Capverdiens, de l'UDC de Fonseca avaient des revendications pour le moins sibyllines, se défendant d'être contre l'indépendance du Cap Vert tout en clamant leur détermination à "ne pas cesser d'être des Portugais" (Cit. in ibid.). Ils réclamaient un référendum sur l'indépendance et, surtout, étaient fermement opposés au plan d'union étroite entre les États capverdien et guinéen, dont la réalisation aurait de fait sonné le glas du rêve de partenariat privilégié et exclusif avec le Portugal.

L'organisation de Fonseca s'était fixée comme objectif d'atteindre le seuil des 5000 adhérents prévu par le projet de loi sur les partis portugais, parcourant à cette fin les milieux ouvriers capverdiens à la recherche de signatures (Presença Cabo-verdiana, 11/74, p. 2). L'UDC portugaise voulait se constituer officiellement en parti afin de devenir, aux yeux de la loi, la seule représentante politique légitime des Capverdiens de la métropole. Dépasser le PAIGC en termes de soutien populaire était une condition essentielle à la réalisation de cette ambition. Les deux mouvements se livraient une bataille dont l'enjeu était le soutien des travailleurs immigrés, moins acquis au PAIGC que les habitants de l'archipel: en effet, ils n'étaient pas touchés par la propagande diffusée quotidiennement dans l'archipel, et, comme ils travaillaient au Portugal, craignaient d'être les premiers à subir les conséquences d'une séparation entre métropole et colonie. Les indépendantistes reconnaissaient que les travailleurs, au contraire de "l'immense majorité" des étudiants et fonctionnaires immigrés, demeuraient un public à conquérir (Cf. Presença Cabo-verdiana, 5/74).

L'UDC avait compris qu'il existait autour de ce thème sensible un moyen de discréditer le PAIGC et appela à l'été 1974 à une mobilisation des travailleurs immigrés. Dans un communiqué intitulé "Notre terre et notre pain en péril", l'UDC accusa le PAIGC d'être responsable de la vague de licenciements qui, depuis le 25 avril, touchait les employés capverdiens de la construction civile, et annonça son intention d'organiser "la plus grande manifestation de Capverdiens jamais vue au Portugal" (Cit. in ibid.; Diário de Lisboa, 19/8/74, p. 4 - ma traduction; Presença Cabo-verdiana, 7/8/74, p. 9). Fonseca, qui revendiquait le soutien des ouvriers du bâtiment, parvint à réunir une centaine d'entre eux lors de la manifestation du 19 
août 1974 à Lisbonne (ibid.). Il affirmait être allé, avec ses militants, à la rencontre de nombreux travailleurs et avoir mesuré leur opposition à l'indépendance totale du Cap Vert ainsi qu'au règne du PAIGC, tout comme leur adhésion à l'idée d'un État indépendant associé au Portugal (Novo Jornal de Cabo Verde', 22/8/74, p. 8).

D'après le PAIGC, les militants de l'UDC profitaient plutôt du déficit d'information des ouvriers pour calomnier le mouvement indépendantiste. Ils auraient colporté la rumeur que l'indépendance forcerait tous les émigrés à rentrer au Cap Vert, ou encore du licenciement professionnel de quiconque refuserait d'adhérer au PAIGC (Presença Cabo-verdiana, 12/74, pp. 2, 6). Les représentants indépendantistes accusaient aussi les "traîtres" du parti adverse d'être de mèche avec les patrons du BTP', d'avoir obtenu d'eux des congés pour leurs employés afin qu'ils puissent participer à la manifestation de l'UDC, et gratifié de 300 escudos les travailleurs qui s'y étaient rendus (ibid., 7/8/74, p. 9; 11/74, p. 2).

La manifestation se termina par un véritable affrontement. 300 à 400 partisans du PAIGC vinrent empêcher les orateurs de l'UDC de prendre la parole en couvrant leurs mots des cris de "Unidade Guiné - Cabo Verde" ou encore "PAIGC, PAIGC", tentant aussi de convaincre les participants ouvriers qu'ils avaient été dupés et manipulés par des colonialistes (ibid., 7/8/74, p. 9). La confrontation se termina par des coups et jets de pierre (A Capital, 19/8/74, p. 13; Novo Jornal de Cabo Verde, 22/8/74, p. 8). À l'issue des événements, le PAIGC affirmait pouvoir apporter les preuves que cette violence avait été préméditée de longue date par les partisans de l'UDC, et mettait en cause la passivité de la police portugaise le 19 août, complaisante selon lui avec les forces "fascistes" et répressive à l'égard des "anticolonialistes" (Presença Cabo-verdiana, 7/8/74, p. 9; A Capital, 19/8/74, p. 13).

\section{Les partis politiques portugais}

Sur le terrain politique, seuls l'UDC, le PAIGC et le GADCG semblaient avoir la prétention de représenter les Capverdiens du Portugal. Les organisations portugaises, elles, feignaient d'ignorer leur existence. Pourtant, l'heure était à la fraternité internationaliste, à la réconciliation entre l'Europe et l'Afrique et à un certain intérêt des médias pour l'actualité des colonies. Les partis de gauche ne cessaient d'afficher leur sympathie pour les mouvements de libération et de s'insurger contre l'oppression des populations africaines (Diário Popular, 7/7/75, p. 9).

La solidarité avec les Africains résidant en métropole était invisible dans cette vague de sympathie. Alors que le sort des émigrés portugais était une préoccu-

\footnotetext{
${ }^{6}$ Principal hebdomadaire capverdien.

7 Secteur du Bâtiment et des Travaux Publics.
} 
pation centrale, celui des Capverdiens au Portugal n'entrait pas en compte dans la gamme d'urgences sociales abordées par la gauche portugaise. Ce n'était pas faute de ne pas savoir: le phénomène était médiatisé, des instituts de réflexion avaient contribué à alerter le monde intellectuel de son existence (Nôs Luta, 26/12/74, pp. 5-6). Le GADCG disposait de nombreux militants actifs au sein des partis portugais et menait un travail de sensibilisation auprès des organisations progressistes du pays (Diário Popular, 17/6/74, p. 9). Le Parti Communiste (PCP) avait bénéficié de contacts avec des membres du PAIGC portugais avant la révolution, formant certains d'entre eux et les soutenant matériellement dans leur lutte clandestine: il est peu probable que ceux-ci n'aient jamais évoqué auprès des communistes la situation des travailleurs immigrés. N'oublions pas que le Parti disposait de multiples relais dans des milieux professionnels où les Capverdiens étaient nombreux. Le PCP comme le Parti Socialiste (PS) étaient bien implantés sur les chantiers navals; des travailleurs capverdiens y furent d'ailleurs abordés et participèrent à des réunions politiques (Cf. Monteiro, 1995, p. 22). Le silence des partis est d'autant plus étonnant qu'en 1974 fut perpétrée une série de crimes racistes à l'encontre des travailleurs capverdiens, face à laquelle la police fit preuve d'une passivité controversée (Diário de Notícias, 18/6/74, p. 8). Loin d'alarmer les politiques, ces évènements furent accueillis par un silence complet.

Pour expliquer ce silence, il n'est pas absurde d'avancer une hypothèse d'ordre électoraliste: celle de la faible sympathie de la clientèle ouvrière des partis de gauche à l'égard de l'immigration. Sur les lieux d'entreprise, certains comportements hostiles étaient patents, et les déclarations des représentants syndicaux à ce propos ambigus. Toute la responsabilité des discriminations était mise sur le compte des patrons. L'attitude des ouvriers européens vis à vis de leurs homologues africains demeurait un sujet tabou (Cf. Carreira, 1977, p. 146). On peut imaginer que le même malaise prévenait certains partis engagés auprès du prolétariat de prendre position en faveur des immigrés. Seuls des groupes marxistes de dimension marginale prenaient fait et cause pour les Capverdiens (Combate ${ }^{8}$, 5/7/74). A en croire Migrações e Turismo ${ }^{9}$, le thème de l'immigration capverdienne n'était pourtant pas absent des discussions politiques privées: pour le journal, les dissensions politiques généralisées depuis le 25 avril 1974 se projetaient sur les immigrés, entre ceux qui saluaient en eux des compagnons de lutte ayant mis fin au colonialisme, et ceux qui les brocardaient comme des ennemis co-responsables des injustices sociales touchant la classe ouvrière portugaise (Migrações e Turismo (1976-1977), décembre-février, pp. 43-46).

\footnotetext{
8 Journal trotskyste portugais.

9 Journal de la commission épiscopale pour les migrations et le tourisme.
} 


\section{Les syndicats}

Les injustices sociales touchaient pourtant largement les Capverdiens, en particulier dans le secteur sinistré de la construction civile. Mobilisables à merci sous le règne de Caetano, les ouvriers de l'archipel étaient désormais eux aussi concernés par l'acquisition d'un certain nombre de garanties sociales. Leur rentabilité en tant que main-d'œuvre en devint moindre pour les industriels. A cela s'ajoutait l'incertitude quant au statut des migrants, encore indéfini légalement, et le début de l'immigration familiale, autant de variables dont certains patrons ne voulaient plus s'embarrasser. Le Novo Jornal de Cabo Verde s'insurgeait du fait que, "après le 25 avril, lorsqu'une brèche parut s'ouvrir pour de nouvelles perspectives, les patrons paniquèrent et répondirent aux revendications par [...] 5000 licenciements [de Capverdiens] sans juste cause" (26/12/74, pp. 5-6 - ma traduction). Certaines sources affirment qu'à l'été 1975 le taux de chômage avoisina les 50\% dans la communauté capverdienne (Cf. Diário de Lisboa, Supplément). Dans un article du Diário de Lisboa, l'un de ses membres témoignait: "Le chômage, c’est le fléau du Capverdien. Il n'y a pas de construction civile. Ça va mal pour le Capverdien" (Cit. in ibid. - ma traduction). Le 16 janvier 1975, le PAIGC avait dénoncé devant l'ONU la mise au chômage progressive des "40 000 travailleurs capverdiens qu'à partir surtout de 1970 le gouvernement de M. Caetano canalisa vers le Portugal" (Cit. in Presença Cabo-verdiana, 7/8/74, p. 10 - ma traduction). C'est que, comme le disait un ouvrier du Cap Vert à la tribune d'une réunion du GADC, licencier les employés africains sans avis préalable ni indemnités était souvent facile, à cause du manque d'expérience ou d'accès à l'information dont souffraient les migrants à l'intérieur des milieux professionnels (ibid.).

Conscients que les Capverdiens représentaient une partie importante des employés du BTP, le principal syndicat de ce secteur avait prétention à les représenter. En février 1975, l'un de ses délégués soulignait que "le Capverdien" était encore un élément exploité et insulté par les patrons (Cit. in Carreira, 1977, p. 146). Il dénonçait les abus de ces derniers, notamment dans le cas des immigrés retournant au Cap Vert:

Quand ["le Capverdien”] cherche à retourner chez lui, il prévient généralement le patron à l'avance qu'il a besoin de la somme réglée [avec l'entreprise] pour pouvoir effectuer le voyage [...] Lorsqu'il se prépare à partir, [le patron] répond qu'il n’a pas d'argent et qu'il ne peut le payer. Puis, le problème finit par s'éterniser, à Lisbonne, nécessitant souvent l'intervention du Syndicat. 
Le syndicat du BTP avait dans son programme un chapitre consacré aux "travailleurs migrants". Mais si le texte convenait de la légitimité pour les "travailleurs immigrés au Portugal (surtout les Capverdiens)" à accéder aux mêmes droits que leur homologues portugais et affirmait sa détermination à les défendre selon les mêmes principes, il ne leur consacrait que quelques lignes, l'essentiel des revendications exposées touchant aux travailleurs portugais émigrés (Andaime ${ }^{10}$, 1976, novembre, p. 14). Pourtant, les Capverdiens étaient loin de former une proportion négligeable des employés du bâtiment. Constituant près de la moitié des ouvriers dans certaines entreprises comme la LISNAVE ou la SETNAVE, géants des chantiers navals de Lisbonne et Setúbal (en 1973 ils étaient plus de 600 à travailler sur les chantiers de la SETNAVE) ${ }^{11}$, ils étaient même majoritaires dans des structures plus modestes ${ }^{12}$.

Ils continuaient malgré cela à être considérés comme une minorité passive. Pour les délégués syndicaux "le blanc, [...] plus politisé" devait influencer "le Capverdien, peu connaisseur de la réalité qui l'entoure" (Cit. in Carreira, 1977, p. 146). A partir de ce genre de considérations s'établissait une hiérarchie d'après laquelle les "blancs" géraient les affaires courantes et disposaient de la tribune, quand les "noirs" étaient relégués au rôle de masse syndicale. Dans la revue du syndicat de la construction civile daté de novembre 1976, on peut voir des photographies de réunions syndicales, et si les noirs y sont très nombreux, ils y figurent toujours au second plan, debout au fond des salles (Andaime, 11/74, p. 14). Ces images reflètent la place réelle des Capverdiens dans la vie syndicale, peut-être aussi leur volontaire mise en retrait de structures au sein desquelles ils ne se sentaient pas justement représentés. Un ouvrier du bâtiment particulièrement engagé ne regrettait-il pas en 1974 que le syndicat ne permette aux immigrés "aucune forme de participation"? (Novo Jornal de Cabo Verde, 26/12/74, pp. 5-6).

On peut difficilement légitimer cette mise à l'écart au nom d'une faible conscience syndicale ou encore d'un déficit d'instruction ou de lucidité des Africains face à l'exploitation. Certes, ils avaient souvent moins d'expérience professionnelle, et l'exclusion pratiquée à leur égard en faisait des individus parfois peu au courant de la législation du travail ou du fonctionnement des syndicats. Mais après tout, seuls 45000 des 120000 ouvriers de la construction civile au Portugal étaient syndiqués. Selon une enquête réalisée auprès de 1606 travailleurs de la région de Lisbonne au lendemain du 25 avril, 42,5\% étaient

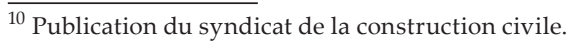

${ }^{11}$ Source :http://www.setubalnarede.pt/content/index.php?action=articlesDetailFo\&rec=6958 [Dernière consultation: 4/5/2011]

${ }^{12}$ Par ex. la papeterie Fernandes. Cf. Nôs Luta, 1/6/75, p. 3.
} 
analphabètes et $17,7 \%$ dotés de la seule instruction primaire: ces chiffres ne sont pas particulièrement inférieurs aux statistiques mesurées par le ministère de l'intérieur chez les émigrants de l'archipel (Vida Mundial, 1975, 20 février, p. 25; Anuário Estatístico (1960-1976), Vol. I \& II). Rien de concret n'indique donc que les Capverdiens étaient moins que les autres ouvriers en capacité de comprendre les rapports de force à l'intérieur de l'entreprise, d'apprendre leurs droits et de mesurer la nécessité de les protéger.

De plus, il semble que les immigrés aient fait preuve d'un désir de participation croissant relativement à la mutation du monde du travail qui prit forme à partir de la révolution des œillets. La presse et les organisations indépendantistes capverdiennes encourageaient vivement les émigrés à prendre toute leur place dans les actions syndicales (Cf. Nôs Luta, 1/5/75, p. 7). Bien vite, une grande partie des Capverdiens commença à voir les syndicats comme des instruments utiles de lutte pour la défense de leurs intérêts (ibid.); utiles et en même temps insuffisamment tournés vers la prise en compte de leurs statut et besoins particuliers de travailleurs immigrés. Partant de cette considération, certains tentèrent d'influer sur la vie syndicale et d'en changer les modalités. Un groupe d'ouvriers de l'archipel co-signa une liste de propositions qu'il transmit, en avril 1975, au syndicat de la construction civile. Cette liste rappelait l'importance numérique des Capverdiens parmi les employés du bâtiment et leur aspiration à la possibilité de se rendre régulièrement au Cap Vert pour régler les questions d'ordre familial. Elle revendiquait, en sus des congés payés, un droit de vacances non rémunérées pouvant aller jusqu'à deux mois par an, avec garantie de réintégration dans l'entreprise, couverture des coûts du voyage par l'entité patronale, et enfin les dispositions de sécurité financières et contractuelles nécessaires pour pouvoir se déplacer inopinément vers leur terre d'origine en cas de problème majeur (ibid.). Ce papier, intitulé "Nous participerons à la vie des Syndicats", témoignait tout à la fois d'une volonté d'être impliqués dans le quotidien de lutte, de négociation et de gestion socioprofessionnelle, et d'être reconnus en leur qualité de travailleurs venus d'ailleurs, touchés par des problèmes spécifiques et nécessitant, le cas échéant, une prise en compte différenciée. Sur la base de cette double ambition, un nombre croissant d'ouvriers capverdiens se mit à rejoindre le syndicat de la construction civile, si bien qu'ils finirent par représenter la moitié de ses effectifs. Mais il fallut attendre 1984 avant que les premiers d'entre eux en intègrent l'équipe de direction (Cf. Monteiro, 1995, p. 43). 


\section{Les collègues portugais}

On peut estimer que le 25 avril marqua le début de l'engagement syndical des Capverdiens, peut-être parce que s'il renforça officiellement leurs droits, il accentua aussi concrètement leur fragilité face aux orientations des patrons de l'industrie, notamment du bâtiment. Ce secteur connut une crise importante lors de la période dite de transition démocratique, résultat entre autres de l'altération radicale des règles de fonctionnement du marché du travail (Cf. Amaro, 1986, pp. 369-372). Une série de réformes engagées dans la foulée du coup d'état révolutionnaire installa dans le paysage professionnel la reconnaissance des droits syndical et de grève, la généralisation des contrats, le contrôle des licenciements, puis encore une nouvelle délimitation du temps de travail et des congés payés plus généreux. Cette mutation entraîna une réaction patronale parfois extrêmement brutale, traduite par le congédiement d'un segment notable de la maind'œuvre et la multiplication de contrats de court terme. Les immigrés firent les frais de cette évolution du BTP, non seulement par le biais de licenciements mais aussi d'une hausse sans précédent de l'embauche au noir, dont les Capverdiens étaient une cible idéale. Le GADCG pointait des mises au chômage massives touchant aussi bien les Capverdiens du bâtiment que ceux des mines ou des chantiers navals. Il accusait le patronat de pratiquer toutes sortes de pressions pour les pousser hors de l'entreprise, en augmentant le prix des baraques dans lesquelles ils étaient logés ou en encourageant les divisions "raciales" parmi les travailleurs (Diário Popular, 17/6/74, p. 9).

Les indépendantistes se plaignaient de ce que la "réaction" désignât les Capverdiens comme responsables du chômage et des bas salaires, répandant ainsi un courant de racisme dans le prolétariat portugais (Nôs Luta, 1/5/75, p. 6, 8). Pour le GADCG, cette manœuvre "postfasciste" dont les patrons étaient les agents, reproduisait le même schéma que celui des conflits militaires d'Afrique contre les mouvements de libération, où la "réaction" avait, "dans son aventure coloniale", fait des travailleurs portugais "des criminels de guerre" (Diário Popular, 17/6/74, p. 9). La comparaison doit être placée dans le contexte de la vive émotion ayant gagné la communauté capverdienne à la suite de certains conflits qui se terminèrent dans le sang. Sur un chantier, en avril 1975, des Capverdiens furent pris à parti par des collègues portugais qui saccagèrent à la pioche leur local d'habitation et assassinèrent deux d'entre eux (Nôs Luta, 1/6/75, p. 6). Un témoin rapporta avoir entendu les agresseurs crier "Vive les Forces Armées ${ }^{13}$. Vive le 25 avril. Pour

\footnotetext{
${ }^{13}$ En référence au Mouvement des Forces Armées (MFA), qui avait piloté la révolution et lancé un programme de réformes très ancré à gauche.
} 
qu'enfin on puisse virer ces negros. C'est [cela], la révolution" (Cit. in Nôs Luta, 1/6/75, p. 6 - ma traduction).

Des évènements d'une ampleur plus grande survinrent en octobre 1976 dans le nord du pays. La Beralt, une compagnie britannique, y exploitait des mines où les conditions de travail étaient à l'origine de tant d'accidents mortels que, dans les années 1950 et 1960, la plupart d'entre eux quittèrent l'entreprise pour aller chercher du travail en France ${ }^{14}$. La Beralt fit appel aux immigrés "ultramarins" pour colmater cette brèche de main-d'oeuvre, jusqu'à en employer environ 600, soit la moitié du personnel total. Dans la période 1974-1976, l'entreprise se divisa en deux blocs, l'un noir, l'autre blanc. L'univers inhumain du travail minier, mais aussi la dégradation des relations des migrants avec la direction de la Beralt qui, cessant de s'intéresser à la main-d'oeuvre capverdienne, avait commencé après la révolution à refuser le renouvellement de certains contrats, exacerbèrent cette atmosphère d'opposition chaque jour plus frontale (Cf. Reis \& Neves, 1979, p. 90). A partir du 5 octobre, de graves incidents opposèrent les mineurs des deux origines, marqués par le lourd bilan d'un mort et de plusieurs blessés graves. L'affaire détériora l'image des immigrés dans l'opinion publique portugaise et choqua le Cap Vert, dont l'ambassade envoya un émissaire sur les lieux pour tenter de régler le conflit. Lors d'une plénière organisée le 7 octobre, l'ensemble des mineurs capverdiens prit la décision d'abandonner les mines aux cris de "Vive l'indépendance" et "Vive le PAIGC" (Cit. in Monteiro, 1995, p. 38). On voit donc que, dans les conflits qui les opposaient, les travailleurs portugais et capverdiens se réfugiaient derrière leurs symboles nationaux respectifs et érigeaient les mots d'ordre révolutionnaires en arguments au milieu de leurs affrontements.

Les mouvements politiques et syndicaux ouvriéristes, aussi bien européens qu'africains, voyaient dans le patronat le principal instigateur de ces troubles. Pour Combate, la réponse aux attaques racistes devait être l'unité de classe et non la division de couleurs (Combate, 5/7/74). Dans certaines entreprises, les ouvriers se montrèrent sensibles à cette idée. Chez J. Pimenta ${ }^{15}$, un plan de suppression de 2000 emplois occupés par des Capverdiens se retrouva en bute à une alliance entre tous les travailleurs, sans distinction de couleur de peau, et dut reculer face à un mouvement que le Novo Jornal identifie comme la première grève du Portugal unissant "blancs et noirs" derrière une cause commune (Novo Jornal de Cabo Verde, 26/12/74, pp. 5-6). Une lutte victorieuse des travailleurs européens et africains, commencée en mai 1974, eût également raison des pratiques contestées de la Papelaria Fernandes, une entreprise employant en majorité des manœuvres

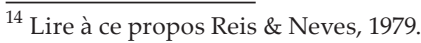

${ }^{15}$ Entreprise phare du secteur du BTP.
} 
originaires de l'archipel et des mineurs de 14 ans, payés très en deçà du salaire déjà peu élevé d'un ouvrier non qualifié (Nôs Luta, 1/6/75, pp. 2-3, 6). Elle s'acheva en mai 1975 par la réalisation des exigences des grévistes, devenant ainsi un symbole d'unité et de communauté d'intérêt entre ouvriers noirs et blancs.

\section{Le problème de la nationalité}

On voit que les Capverdiens durent faire face à la précarité sociale et professionnelle. Mais cette précarité était aussi légale. Après plus d'un an d'incertitude quant au devenir de leur statut, le sort des Capverdiens du Portugal fut scellé avec la confirmation du processus d'indépendance et surtout, le 24 juin 1975, par le décret-loi no 308-A/75. Le texte stipulait que les individus nés en "territoire ultramarin devenu indépendant" perdaient automatiquement la nationalité portugaise, à moins d'avoir des origines portugaises pouvant aller jusqu'au troisième degré ou d'être une femme mariée, veuve ou divorcée d'un Portugais (Cf. Pires, 2003, pp. 126-128). Cette loi marqua profondément l'histoire de l'immigration au Portugal, en entérinant la perception du phénomène comme une menace et en ouvrant une époque de politiques de dissuasion des flux migratoires. Avec le décret-loi 308-A/75, le Portugal s'inscrivait dans une législation de ius sanguinis et limitait les possibilités d'acquérir la nationalité. Il faut préciser que cette loi fut moins pensée pour régler la situation juridique de l'immigration capverdienne que dans le contexte d'arrivée massive de réfugiés portugais jusque là établis dans les colonies. Elle constitua un outil de choix sélectif destiné à limiter essentiellement à la population blanche les flux en provenance d'Afrique (Cf. Pires, 2003, pp. 126-128). Almeida Santos, rédacteur de la loi en tant que ministre de la coordination interterritoriale pour le PS, la décrivit comme une mesure destinée à éviter le débarquement à Lisbonne "d'un million de personnes ou plus" depuis l'Angola, du fait de la situation de guerre civile sévissant dans l'ancienne colonie (Cit. in Pires, 2003, p. 128). Etablissant une barrière entre l'Afrique et l'Europe, le décret modifia clairement la nature du parcours migratoire Cap Vert - Portugal, qui n'allait devenir possible qu'avec une lettre de regroupement ou un contrat de travail préalablement émis (Cf. Lopes Filho, 1980, p. 446). Le décret maintenait partiellement et provisoirement le principe du ius solis, laissant aux individus nés dans les anciennes colonies et résidant depuis plus de cinq ans avant la révolution des œillets sur le territoire portugais la possibilité de conserver la nationalité portugaise (Cf. Pires, 2003, p. 127). Mais cette disposition n'avantageait guère la communauté capverdienne puisque c'est justement dans les cinq dernières années du régime marceliste ${ }^{16}$ que fut accueilli le gros des flux en provenance de

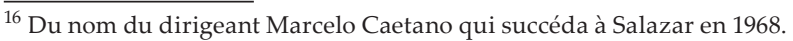


l'archipel. En outre, la conservation de la nationalité portugaise était conditionnée au renoncement à toute autre nationalité, ce qui impliquait de la part des individus demandeurs l'abdication de leur citoyenneté capverdienne (Cf. Ramos, 1976). Globalement, la position des ressortissants de l'archipel, surtout les plus pauvres, se trouva nettement fragilisée par ce nouvel appareil législatif, renforcé par une application stricte du non renouvellement des papiers d'identité et un décret-loi de préférence nationale qui, en 1976, réservait aux seuls citoyens nationaux l'accès aux logements sociaux (Cf. Monteiro, 1995, p. 55; L. Silva, 2007, p. 544). On peut mesurer l'injustice d'une telle mesure étant donné que les immigrés comptaient parmi les plus mal-logés dans les villes du pays.

Il semblerait qu'au-delà de ces nouvelles formes d'exclusion, le basculement des "ultramarins" dans le domaine des "étrangers" contribua à briser un certain nombre de tabous racistes, puisque dans la période de "transition démocratique" les harcèlements, bavures et crimes policiers, les manifestations populaires d'agressivité et les refoulements à la porte des lieux publics se succédèrent à l'encontre des ressortissants de l'archipel, sans que ces évènements ne soient remarquablement relayés par les organes d'information ni dénoncés par les organisations publiques (Diário Popular, 17/6/74, p. 9; Presença Cabo-verdiana, 6/74, p. 2; 10/74, p. 7; Diário de Notícias, 18/6/74, p. 8). Certains interprétèrent cette multiplication des entreprises d'exclusion, comme le salaire de l'indépendance. Ainsi, le GADCG expliquait en juin 1974 que les Capverdiens étaient la cible de licenciements massifs car les patrons voulaient faire d'eux "le bouc émissaire de leur imminente défaite coloniale" (Cit. in Diário Popular, 17/6/74, p. 9). "Vous pouvez avoir l'indépendance; mais très bien, alors vous n'aurez pas de travail, allez dans votre pays" aurait été une phrase-type des patrons pendant la crise de la construction civile (Cit. in ibid. - ma traduction).

Il est vrai que, pour certaines élites portugaises, l'adhésion populaire des Capverdiens aux revendications d'indépendance émises par le PAIGC fut une surprise qui laissa un goût amer dans la bouche de quelques-uns, convaincus que le Cap Vert, en vertu de sa proximité culturelle avec le Portugal, ne suivrait pas la route des autres colonies africaines. Mário Soares confiera quelques décennies plus tard avoir été de ceux-là. "Sincèrement" dira-t-il, "j'ai toujours été convaincu que le Cap Vert avait plus à gagner comme région [...] associée au Portugal, que comme [...] pays africain. Le Cap Vert, ce n'est pas vraiment l'Afrique" (Cit. in Lopes, 2002, p. 320 - ma traduction). Or, à en croire Luiz Silva, Soares insista personnellement, en tant que ministre des affaires étrangères de mai 1974 à mars 1975, pour que la mesure de non renouvellement des passeports portugais s'applique également aux Capverdiens émigrés dans des pays tiers (France, 
Luxembourg, Suisse, Hollande ou Italie) faisant tomber des milliers d'entre eux dans la clandestinité, et les privant ainsi de tout ou partie des services de protection sociale (Cf. 2003, p. 22). Cette politique participa d'un ensemble de mesures dans le cadre desquelles les immigrés apparurent comme les sacrifiés de la décolonisation.

\section{La nation capverdienne}

La décolonisation, pourtant, avait provoqué une vague d'enthousiasme chez les travailleurs immigrés. Certains d'entre eux purent assister, à Praia, à la cérémonie d'indépendance, grâce à un avion affrété par les organisations capverdiennes de Hollande, qui fit escale au Portugal (ibid.). Les autres furent nombreux à se joindre aux festivités organisées par les GADCG ou à manifester leur euphorie dans les rues de Lisbonne (Diário Popular, 7/7/75, p. 9). Pour la première fois, dans la deuxième partie de l'année 1975, les flux de déplacement du Portugal vers l'archipel devinrent supérieurs à ceux des migrations Cap Vert - Portugal (Anuário Estatístico (1960-1976), Vol. I \& II). Il est envisageable que cette spectaculaire inversion de tendance eût correspondu à une volonté de retour au pays de la part des migrants pour participer à la construction du nouvel État indépendant, comme le souhaitait le PAIGC. Le parti n'avait pas ménagé ses efforts pour capter la sympathie des travailleurs émigrés. Le président du Cap Vert se rendit à Setúbal pour leur promettre de résoudre leurs problèmes (Novo Jornal de Cabo Verde, 17/4/75, p. 3). Mais il les appela en même temps à investir dans les programmes de construction économique du nouveau régime. Ceci montre que le gouvernement du Cap Vert avait autant besoin de l'argent des émigrants que ces derniers ne nécessitaient le soutien politique du gouvernement (Presença Caboverdiana, 5/6/75, p. 3). Pour Nardi Dias de Sousa, la dépendance des îles à l'émigration, héritée en partie de la situation coloniale, conduisit le PAIGC à considérer les émigrés de la même manière que ne l'avait fait l'État colonial: comme une force de travail plutôt que comme des porteurs de richesse culturelle et une partie intégrante de la nation (2003, p. 109). Il affirme que le Cap Vert négocia avec le Portugal la poursuite de l'émigration, donnée perceptible dans les accords signés entre les deux pays (Diário Popular, 7/7/75, p. 9), et va jusqu'à dénoncer un "complot", fomenté, au nom de leurs intérêts économiques respectifs, par les deux États sur le dos des migrants: alléger, au Cap Vert, le fléau du chômage interne; continuer, au Portugal, à occuper les emplois des secteurs d'activité délaissés par les classes populaires émigrées vers le reste de l'Europe. 
Finalement le PAIGC, d'après Dias de Sousa et d'autres chercheurs et essayistes expatriés ${ }^{17}$, alimenta volontairement la croissance de toute une classe d'émigrés afin de bénéficier de leurs envois d'argent, tout en faisant officieusement le nécessaire pour qu'ils ne demeurent pas des citoyens capverdiens à part entière. Le risque était trop grand que des individus vivant dans des démocraties pluralistes où la censure d'État n'existait pas sous des formes aussi restrictives qu'au Cap Vert - comme les États-Unis, la France ou le Portugal d'après 1974 - n'adhèrent pas au régime de parti unique et de répression politique mis en place par les héritiers de $\mathrm{Cabral}^{18}$. Cette non-acceptation d'une partie des émigrés dans la nouvelle communauté nationale se manifesta à travers la négation partielle de leur citoyenneté, notamment en ce qui concerne le droit de vote. Lors des premières élections de l'assemblée nationale capverdienne, qui eurent lieu à l'été 1975, ce droit fut réservé aux personnes résidant à l'étranger depuis moins de cinq ans, à moins que ces derniers n'eussent à leur charge des enfants mineurs de moins de 17 ans ou/et un(e) conjoint(e) vivant(e) encore dans l'archipel, lié à l'intéressé sous le régime juridique de non séparation des biens (Barros, 2001, pp. 121-122; Cf. aussi Novo Jornal de Cabo Verde, 24/4/75, p. 7): en clair, la pleine jouissance de la citoyenneté capverdienne était réservée à ceux dont l'envoi d'espèces en Afrique était, en raison de connexions familiales, quasiment assurée. Renato Cardoso, membre de la Commission Électorale, justifiait officiellement cette règle au nom de motifs culturels, arguant du fait que les émigrés partis "depuis longtemps"19 n'avaient plus de lien avec les îles ni de connaissance des problèmes actuels du pays (Cit. in Novo Jornal de Cabo Verde, 24/4/75, p. 7). Pourtant, les études sociologiques démontrent la subsistance, souvent sur plusieurs générations, de liens forts des émigrés capverdiens du Portugal avec leur pays d'origine et d'une invariable volonté de retour, planifié à moyen ou long terme (Cf. Batalha, 2004; Gomes, 1999; Saint-Maurice, 1997; Semedo, 2005). Sans compter que l'émigration était l'un des principaux enjeux du développement politique, économique et social d'une terre dont la moitié des ressortissants vivait déjà à l'étranger. Au Portugal, les services consulaires du gouvernement PAIGCiste organisèrent les élections un samedi, jour complet de travail pour la plupart des travailleurs immigrés (Diário de Lisboa, 21/6/75, p. 7). Le résultat fut donc très limité. Au total, seuls 2 ou 3\% de la population émigrée totale votèrent à l'étranger lors de ces élections (ibid., 1/7/75, p. 7). Ceci était sans doute dû aussi bien aux obstacles posés par le PAIGC

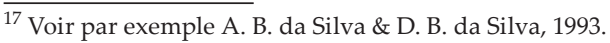

${ }^{18}$ Amílcar Cabral, assassiné en 1973, était le leader historique du PAIGC.

${ }^{19}$ On laissera à son auteur la responsabilité de cette appréciation, cinq années - durant lesquelles, qui plus est, le Cap Vert n'existait pas en tant qu'État - pouvant aussi apparaître comme une période courte.
} 
sur la route des émigrés aspirant à la citoyenneté qu'au désintérêt de ces derniers pour un scrutin où les candidats étaient tous présentés par le même parti. Selon Luiz Silva, les décisions prises "à la légère" au préalable des premières élections eurent un effet catastrophique sur le crédit du PAIGC auprès des émigrés, qui se sentirent exclus du processus d'indépendance, auquel ils considéraient pourtant avoir pris leur part, notamment en améliorant la situation économique du pays et, pour certains d'entre eux, en se battant à l'intérieur de la métropole contre le régime marceliste (Cf. 2007, pp. 543-546).

$\mathrm{Au}$ lieu de reconnaître cette contribution, le parti nationaliste préférait s'attaquer à ces expatriés estrangeirados qui, selon lui, s'étaient compromis dans le colonialisme et avaient quitté les îles pour le Portugal par peur de l'avènement d'un régime indépendantiste et socialiste (Cf. Batalha, 2004, p. 102). Le mot était, il est vrai, destiné à la bourgeoisie et non aux "petits" immigrés économiques, mais il resta dans les esprits et son utilisation populiste portait en elle les germes d'un amalgame avec toutes les couches de l'émigration. En outre, après 1974, bon nombre des anciens "résistants" capverdiens expatriés dans l'ancienne métropole et qui avaient joué un rôle important dans la lutte contre le colonialisme furent mis à l'écart par le parti en raison de leurs positions politiques dissidentes, comme leur soutien au principe de démocratie pluraliste (Cf. Raimundo, 2008, p. 72). Avec leur marginalisation, toute une partie de la contribution des émigrés au mouvement de libération fut ignorée par le nouvel État.

Il faut dire que l'émigration, considérée comme l'héritage d'une histoire douloureuse, conséquence de la pauvreté et de l'exploitation, était officiellement indésirable et souvent dépeinte comme le résultat d'une machination colonialiste. Dans ce contexte, son développement représentait un échec pour le PAIGC, censé débarrasser le pays des fléaux de l'époque salazariste. Cabral lui-même n'avait-il pas proclamé vouloir libérer l'archipel de l'émigration (Cf. Barbe, 2003, p. 130)? Prenant ses préceptes au pied de la lettre, les dirigeants qui construisirent la nation capverdienne prirent le risque de culpabiliser les émigrants. En déplacement à Lisbonne, le président du Cap Vert insista ainsi sur l'idée que si pendant longtemps, les Capverdiens avaient pensé que l'émigration était la solution à leurs problèmes, il était désormais temps de construire les îles depuis l'intérieur (Presença Cabo-verdiana, 5/6/75, p. 3). C'est en ce sens que le parti appela en 1975 les émigrés à regagner le pays pour venir participer à sa construction, comme si la pleine citoyenneté n'était possible qu'à l'intérieur des îles. C'était passer à côté de la particularité de la nation capverdienne, dont de plus en plus de chercheurs estiment qu'elle a construit sa richesse et s'est solidifiée grâce à sa dimension 
transnationale et aux réseaux intercontinentaux constitués par l'enchevêtrement des diverses chaînes migratoires dont elle fut la source ${ }^{20}$.

\section{Conclusion}

Les immigrés capverdiens du Portugal contribuèrent aux changements qui soulevèrent l'espace lusophone au lendemain de l'ère coloniale. Leur activité au sein de la société portugaise constitua en soi une remise en question de la doctrine coloniale de stricte dualité entre métropole et colonies. Mais leur devenir après 1974 démontre aussi les failles d'un processus de décolonisation mentalement incomplet quoique officiellement effectué. Leur histoire témoigne de la subsistance de réflexes systématisés pendant la période de la dictature: la colonisation "interne" se poursuivit malgré la disparition du statut de colonisé. Encore plus précarisés car juridiquement déclassés par la perte de la nationalité portugaise, les travailleurs capverdiens continuèrent à jouer le rôle de la maind'œuvre coloniale.

Toutefois, leur arrivée changea le visage des périphéries urbaines et constitua un facteur de transformation des relations sociales. De société coloniale, le Portugal devenait lentement une société multiculturelle. La mutation n'est peutêtre pas encore achevée. Aujourd'hui pourtant, les migrants capverdiens et leurs descendants sont de plus en plus nombreux à réclamer reconnaissance et droit de participation, non seulement à la vie sociale et politique portugaise, mais aussi à l'évolution de la nation capverdienne, devenue une démocratie libérale plus que jamais dopée par l'importance de sa communauté émigrée, éparpillée aux quatre coins du monde.

Ces revendications peuvent s'appuyer sur l'expérience de la première génération de migrants ouvriers, qui connurent le Portugal et le Cap Vert avant et après la décolonisation et dont le travail, les économies et l'apport culturel représentèrent une contribution importante, bien que trop souvent ignorée, dans la construction de sociétés post-coloniales.

\section{Références}

Acker, A. (2009). L'intégration des migrants capverdiens dans un Portugal en transition (19681976). Mémoire de Master non publié, Paris, Université Paris VII - Denis Diderot.

A Capital, Lisbonne (1974), 19 août.

Amaro, R. R. (1986). Mercado de trabalho e franjas marginalizadas: O caso dos imigrantes cabo-verdianos. In CISEP (Ed.), O comportamento dos agentes económicos e a reorientação da política económica, II Conferência do CISEP, Vol. I. Lisbonne: Instituto Superior de Economia, 355-378.

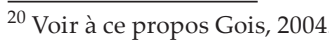


Andaime, Lisbonne (1976), novembre.

Barbe, A. (2003). Les îles du Cap Vert de la découverte à nos jours: Une introduction. Paris: L'Harmattan.

Barros, J. M. B. de (2001). A integração dos emigrantes no sistema político caboverdiano. Lisbonne: ISCTE.

Batalha, L. (2004). The Cape Verdean diaspora in Portugal. Colonial subjects in a postcolonial world. Lanham: Lexington Books.

Carita, C., \& Rosendo, V. N. (1993). Associativismo cabo-verdiano em Portugal. Estudo de caso da Associação Cabo-verdiana em Lisboa. Sociologia - Problemas e Práticas, 13, pp. 135-152.

Carreira, A. (1977). Migrações nas ilhas de Cabo Verde. Lisbonne: UNL.

Constituição da República Portuguesa. Lei do Tribunal Constitucional (2005).

Combate, Lisbonne (1974), 5 juillet.

Diário de Lisboa, Lisbonne (1974), 16 août. Aussi: 19 août; (1975) 21 juin, 1er juillet (Supplément), 5 juillet.

Diário de Notícias, Lisbonne (1974), 18 juin.

Diário Popular, Lisbonne (1974), 17 juin. Aussi: (1975) 7 juillet.

Gois, P. (2004). A construção secular de uma identidade transnacional: A cabo-verdianidade do (ou no) mundo cabo-verdiano. Projet de doctorat, Universidade do Porto [http://www. oi.acidi.gov.pt/docs/pdf/Projecto\%20Phd\%20PGois.pdf].

Gomes, I. B. (Ed.) (1999). Estudo de caracterização da comunidade caboverdeana residente em Portugal. Lisbonne: Embaixada de Cabo Verde.

Anuário Estatístico (1960-1976), Vol. I \& II. Lisbonne: Instituto Nacional de Estatística.

Lopes, J. V. (2002). Cabo Verde. Os bastidores da independência. Praia: Spleen.

Lopes Filho, J. (1980). O emigrante cabo-verdiano em Lisboa. África. Literatura, Arte e Cultura, 9, pp. 444-453.

Migrações e Turismo, Lisbonne (1976-1977), décembre-février.

Monteiro, V. (1995). Portugal crioulo. Praia: ICL.

Nôs Luta, Lisbonne (1975), 1er juin. Aussi: 1er janvier, 1er mai.

Novo Jornal de Cabo Verde, Praia, Cap Vert (1974), 22 août. Aussi: 26 décembre; (1975) 17 avril, 24 avril.

Pires, R. P. (2003). Migrações e integração: Teoria e aplicações à sociedade portuguesa. Oeiras: Celta.

Presença Cabo-verdiana, Lisbonne (1974), mai. Aussi: juillet-août, octobre, novembre, décembre; (1975), mai-juin.

Raimundo, G. (2008). Sôdade de Cabo Verde. Lisbonne: G. Raimundo.

Ramos, R. M. M. (1976). Nacionalidade e descolonização. Algumas reflexões a propósito do decreto-lei no 308-A/75 de 24 de Junho. Revista de Direito e Economia, 1, pp. 121-151.

Reis, D., \& Neves, F. P. (1979). A guerra da mina: Os mineiros da Panasqueira. Lisbonne: Regra do Jogo.

Rocha, E., Agualusa, J. E., \& Semedo, F. (1993). Lisboa africana. Lisbonne: Asa.

Saint-Maurice, A. de (1997). Identidades reconstruídas: Cabo-verdianos em Portugal. Oeiras: Celta. 
Semedo, M. E. B. (2005). O retorno de emigrantes e seus impactes na economia cabo-verdiana: Um estudo de caso. Lisbonne: ISCTE.

Silva, A. B. da, \& Silva, D. B. da (Eds.). (1993). A odisseia crioula: As tristezas, alegrias e esperanças do emigrante cabo-verdiano. Oslo: Alpha Beta Sigma.

Silva, L. (2003). A juventude emigrante caboverdiana em França e a lusofonia. Latitudes, 18, pp. 21-23.

Silva, L. (2007). Reflexões de um emigrante sobre os trinta anos da Independência de Cabo Verde. Direito e Cidadania, III, pp. 539-556.

Sousa, N. D. de (2003). Imigração e cidadania local: Associativismo imigrante e políticas públicas de Portugal. Praia: Instituto da Biblioteca Nacional e do Livro.

Teixeira de Sousa, H. (1994). Entre duas bandeiras. Mem Martins: Europa-América.

Vaz, J. M. (1972). Os caboverdianos em Lisboa. Lisbonne: LIAM.

Vida Mundial, Lisbonne (1975), 20 février. 